\title{
Die KN als internationale Fachzeitschrift?! Ein Blick auf aktuelle Entwicklungen (2019-2021)
}

\author{
Dennis Edler ${ }^{1}$
}

Accepted: 15 February 2022 / Published online: 22 February 2022

(c) The Author(s) 2022

Liebe Leserinnen und Leser,

mit diesem Heft starten die KN - nachdem bereits einige wissenschaftliche Fachartikel sowie der KN-Info und Praxisteil (KNIP) als Publikationen (Online First und Open Access) erschienen - in den 72. Jahrgang. Dank des Datenrepertoires von Springer Nature, aus dem regelmäßig Statistik und Grafiken durch den Senior Journal Development Editor des Verlags abgeleitet und in Online-Meetings mit der KN-Redaktion diskutiert werden, ist es möglich die Entwicklung der KN für die letzten drei Jahre - anhand ausgewählter Beispiele - zu veranschaulichen.

Bei einer Betrachtung der Zugriffe (,Total Item Requests") auf die online veröffentlichten KN-Fachartikel zeigt sich eine deutliche Steigerung der Zahlen, im Betrachtungszeitraum 2019 bis 2021 (Abb. 1). Auf Jahresebene zusammengefasst kommen die $\mathrm{KN}$ auf Zugriffszahlen, die sich seit 2019 annähernd verfünffacht haben (2019: 17.114; 2020: 47.842; 2021: 84.507). Die hohe digitale und internationale Sichtbarkeit des gewechselten Verlagshauses sind sicherlich ein ausschlaggebender Grund für die zunehmende Verbreitung des wissenschaftlichen Outputs der KN. Hinzu kommt, dass ab dem ersten Heft des 2019er Jahrgangs die meisten Fachartikel als Open Access Publikationen erschienen. Über die betrachteten Jahre stieg die Zahl dieser frei abrufbaren Artikel (2019: 15; 2020: 15; 2021: 20). Auch der Aufbau des Online-Archivs vorausgehender Jahrgänge mag zu den steigenden Zugriffszahlen beitragen.
Die aktuelle internationale Sichtbarkeit der KN wird durch die prozentuale Verteilung der Online-Zugriffsländer (Abb. 2) deutlich. Dabei zeigt sich, dass aus den mit den KN eng verbundenen deutschsprachigen Ländern Deutschland, Österreich und Schweiz im vergangenen Jahr ca. 37 Prozent der Online-Zugriffe stammen. Im Umkehrschluss entstehen etwa zwei Drittel der Online-Zugriffe außerhalb dieser Länder, u.a. in Indien (15\%) und in den USA (7\%). Das Tortendiagramm (in Abb. 2) vermittelt einen guten visuellen Eindruck dazu, dass annähernd die Hälfte der OnlineZugriffszahlen (2021) auf die wissenschaftlichen Fachartikel auf Länder außerhalb Europas zurückgehen.

Diese kurze Vorstellung aktueller Statistiken zur jüngsten Entwicklung der KN, die das Team von Springer Nature der KN-Redaktion dankenswerterweise zur Veröffentlichung im Editorial zur Verfügung gestellt haben, deuten auf einen ,großen Sprung “ der KN - hin zu einer breiteren und internationalen Leserschaft. Zwar ist die Messgröße „Item Request “ bzw. Online-Zugriffszahl primär gebunden an erfasste Klicks auf der KN-Webseite (Artikelaufruf und Download) und nicht zwangsläufig gekoppelt an das tatsächliche Lesen und inhaltliche Verarbeiten eines Aufsatzes, jedoch spricht die Entwicklung dafür, dass Artikel gut erreicht werden können und weltweit gesucht werden. Vor diesem Hintergrund sind die KN auf einem guten Weg sich stärker als internationale Fachzeitschrift, die Wissenschaft und Praxis gleichermaßen bedient, zu etablieren - diesen Weg sollten wir weitergehen!
Dennis Edler

Dennis.Edler@ruhr-uni-bochum.de

1 Bochum, Germany 


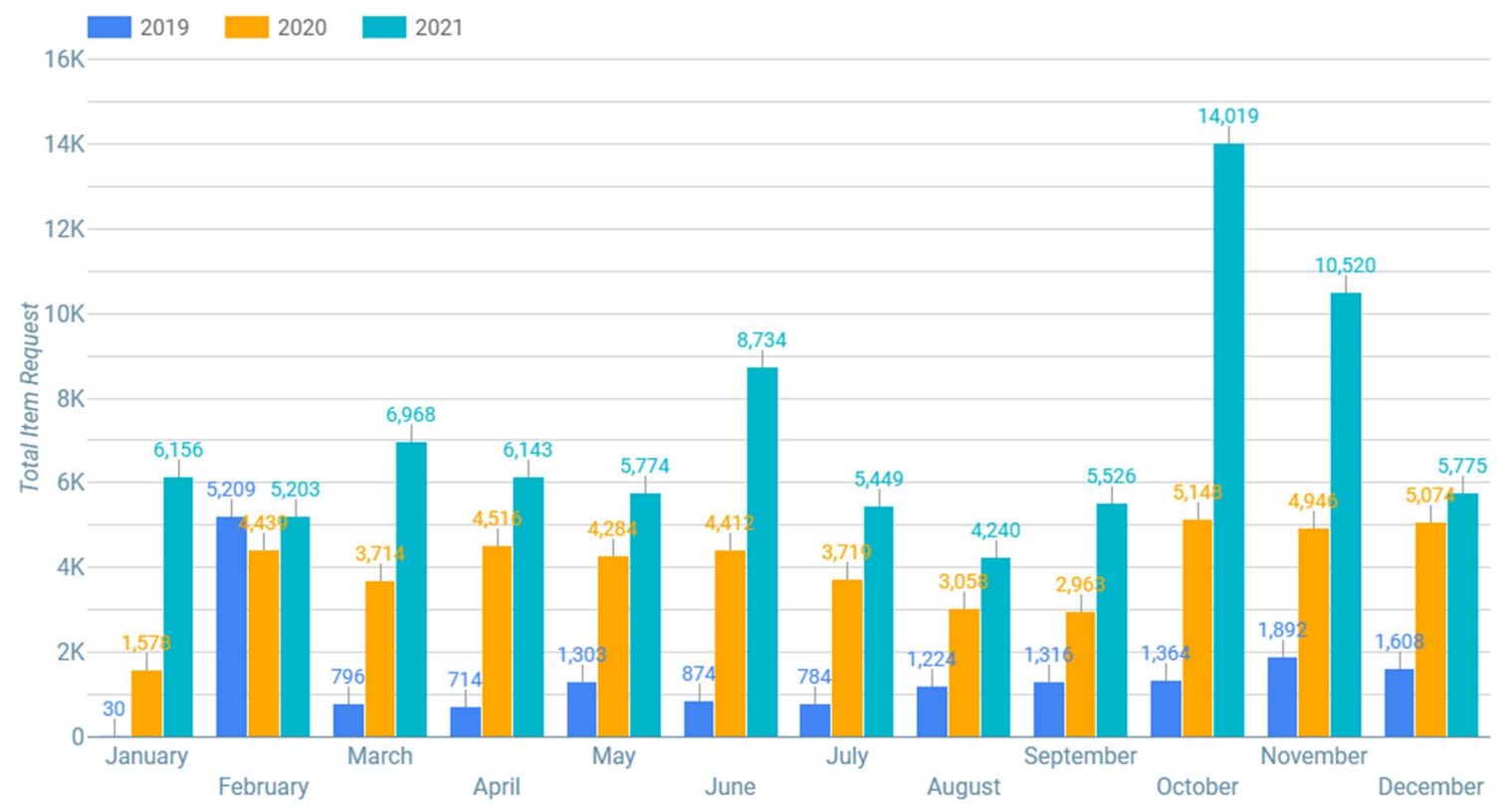

Abb. 1 Online-Zugriffe auf KN-Artikel (2019-2021) [ Quelle: Google Analytics data on Google Big Query]

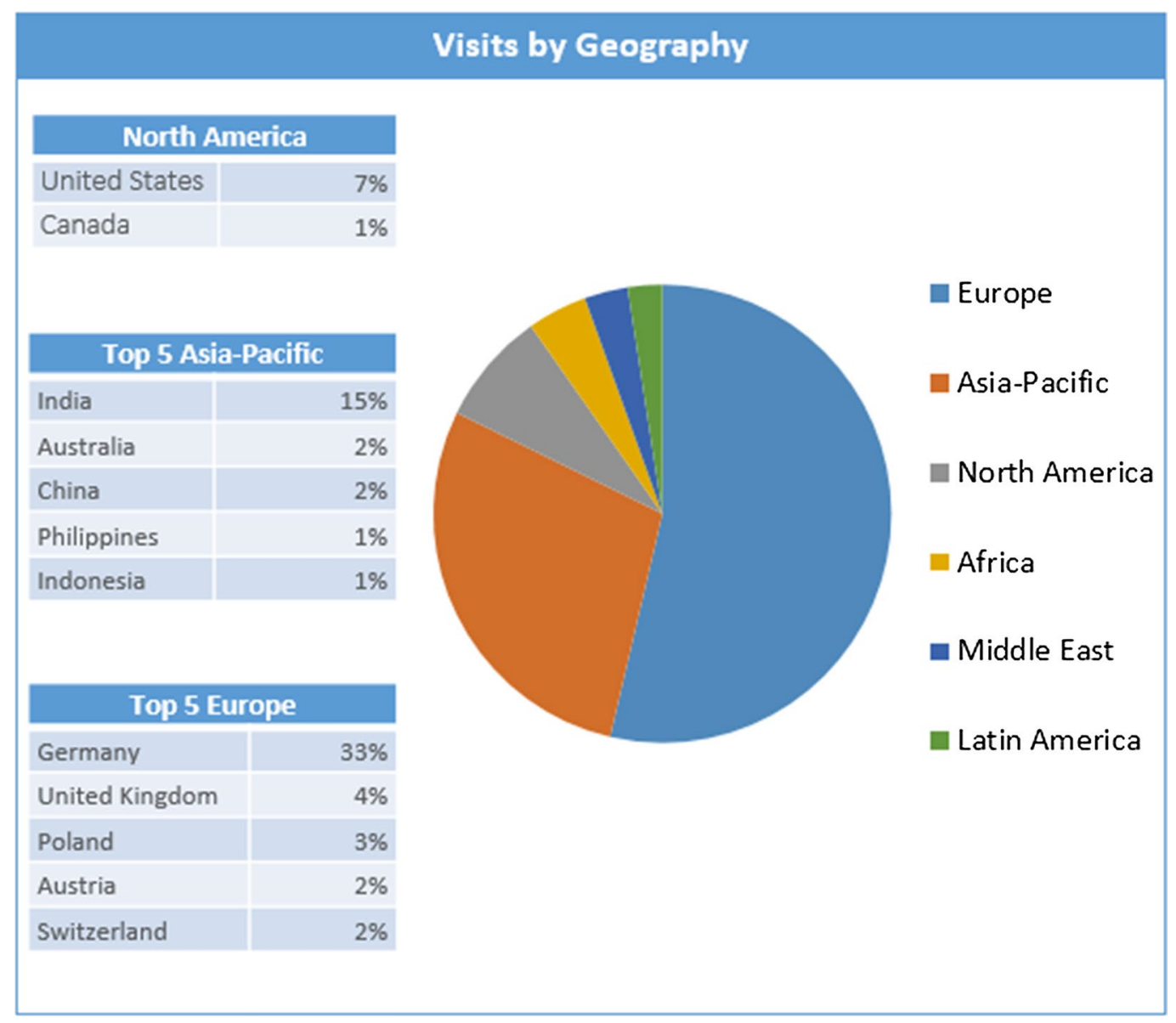

Abb. 2 Verortete Online-Zugriffe (2021) [ Quelle: Google Analytics data on Google Big Query] 
Ich wünsche Ihnen und Euch viel Spaß beim Lesen der wissenschaftlichen Artikel und des umfangreichen KN-Infound Praxisteils!

Herzliche Grüße - im Namen der gesamten KN-Redaktion.

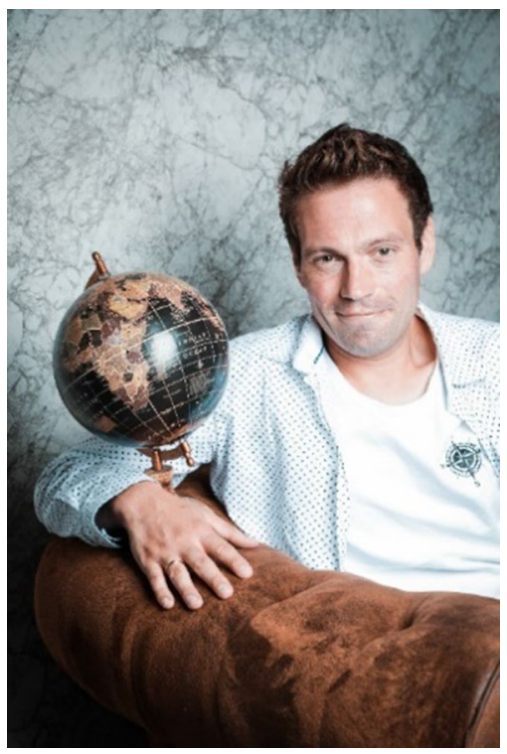

Dennis Edler
Open Access This article is licensed under a Creative Commons Attribution 4.0 International License, which permits use, sharing, adaptation, distribution and reproduction in any medium or format, as long as you give appropriate credit to the original author(s) and the source, provide a link to the Creative Commons licence, and indicate if changes were made. The images or other third party material in this article are included in the article's Creative Commons licence, unless indicated otherwise in a credit line to the material. If material is not included in the article's Creative Commons licence and your intended use is not permitted by statutory regulation or exceeds the permitted use, you will need to obtain permission directly from the copyright holder. To view a copy of this licence, visit http://creativecommons.org/licenses/by/4.0/. 